\title{
Are blood pressure level and variability related to pregnancy outcome? Analysis of control of hypertension in pregnancy study data
}

\author{
Laura A Magee $^{\mathrm{a}, *}$, Joel Singer ${ }^{\mathrm{b}}$, Terry Lee ${ }^{\mathrm{c}}$, Richard J McManus ${ }^{\mathrm{d}}$, Sarah Lay-Flurrie ${ }^{\mathrm{d}}$, \\ Evelyne Rey ${ }^{\mathrm{e}}$, Lucy C Chappell ${ }^{\mathrm{a}}$, Jenny Myers ${ }^{\mathrm{f}}$, Alexander G Logan ${ }^{\mathrm{g}}$, Peter von Dadelszen ${ }^{\mathrm{a}}$ \\ a Department of Women and Children's Health, King's College London, UK \\ ${ }^{\mathrm{b}}$ School of Population and Public Health, Centre for Health Evaluation and Outcome Science, Providence Health Care Research Institute, University of British Columbia, \\ Vancouver, Canada \\ ${ }^{\mathrm{c}}$ Centre for Health Evaluation and Outcome Science, Providence Health Care Research Institute, University of British Columbia, Vancouver, Canada \\ ${ }^{\mathrm{d}}$ Nuffield Department of Primary Care Health Sciences, University of Oxford, UK \\ ${ }^{\mathrm{e}}$ Departments of Medicine and Obstetrics and Gynaecology, Université de Montreal, Canada \\ ${ }^{\mathrm{f}}$ Division of Developmental Biology and Medicine, Manchester Maternal \& Fetal Health Research Centre, UK \\ ${ }^{g}$ Department of Medicine, University of Toronto, Canada
}

\section{A R T I C L E I N F O}

\section{Keywords:}

Blood pressure

Variability

Pregnancy outcome

\begin{abstract}
A B S T R A C T
Objective: To examine the relationship between pregnancy outcomes and BP level and variability.

Design: Secondary analysis of CHIPS trial data (Control of Hypertension In Pregnancy Study, NCT01192412).

Setting: International.

Population or sample: Women with chronic or gestational hypertension.

Methods: BP measurement was standardised in outpatient clinics. Adjusted (including for allocated group) mixed effects logistic regression was used to assess relationships between major CHIPS outcomes and both BP level (mean of clinic readings) and visit-to-visit within-participant BP variability (standard deviation and average real variability of absolute successive difference of BP values). BP values 7-28 days prior to outcomes (or birth for perinatal outcomes) were excluded in sensitivity analyses.

Main outcome measures: Major CHIPS outcomes.

Results: Among 961 (97.4\%) women, higher BP level was associated with more adverse maternal and perinatal outcomes (usually at p < 0.001) except for serious maternal complications. Among 913 (92.5\%) women with at least two post-randomisation outpatient visits, higher BP variability was associated with increased odds of severe hypertension and pre-eclampsia (usually at $\mathrm{p}<0.01$ ). Sensitivity analyses suggested reverse causality for these maternal outcomes, but greater diastolic BP variability may have been associated with fewer adverse perinatal outcomes.

Conclusions: Higher BP is an adverse prognostic marker, regardless of target BP. While the association between higher BP variability and severe hypertension and pre-eclampsia may be related to higher BP at diagnosis, our results suggest a possible advantage of BP variability for the fetus, through undefined mechanisms.

Tweetable abstract: Higher blood pressure (BP) is associated with more adverse pregnancy outcomes, but higher BP variability may be good for the baby.
\end{abstract}

\section{Introduction}

Blood pressure (BP) level has long been associated with elevated cardiovascular risk [1]. Most investigators have studied BP measurements taken in office/clinic settings, although similar (or stronger) associations have been found when BP has been recorded at home (by the patient) or while ambulatory (usually over a $24 \mathrm{hr}$ period) [2]. There is consensus that persistent hypertension, defined as BP values $\geq 140$ / $90 \mathrm{mmHg}$ in the office (or $\geq 135 / 85 \mathrm{mmHg}$ out-of-the-office) should be addressed with lifestyle change and/or antihypertensive treatment to improve cardiovascular outcomes in the long-term, with American multidisciplinary guidelines advocating an even lower threshold for

\footnotetext{
* Corresponding author at: Department of Women and Children's Health, School of Life Course Sciences, King's College London, Becket House, 1 Lambeth Palace Road, London SW1 7EU UK.

E-mail address: Laura.A.Magee@kcl.ac.uk (L.A. Magee).
} 
intervention [3-5].

Hypertension in pregnancy is defined as a systolic BP (sBP) $\geq 140 \mathrm{mmHg}$ and/or diastolic BP (dBP) $\geq 90 \mathrm{mmHg}$ [6]. While BP normally falls in pregnancy, reaching a nadir of up to $5 \mathrm{mmHg}$ lower at about 20 weeks, and then climbing towards pre-pregnancy levels by term, there is a continuous relationship between higher BP and worse maternal outcomes among women with any type of pregnancy hypertension, as well as those with pre-eclampsia specifically [7-10]. Severe elevations of BP (to $\geq 160 / 110 \mathrm{~mm} \mathrm{Hg}$, regardless of the hypertensive disorder) are also associated with adverse maternal and perinatal outcomes [11].

Outside of pregnancy, we know that hypertension over decades leads to endothelial damage and an increased risk of cardiovascular disease, particularly stroke $[12,13]$. Cardiovascular risk is mitigated by the slow onset of hypertension that allows for some adaptation [14]. Diastolic (rather than systolic) hypertension is particularly important in mediating adverse effects in young adults. While the actual level of BP is very important, we now know that over and above this, variability in $\mathrm{BP}$ has an independent effect on cardiovascular risk [15]. BP variability reflects the integrated effects on BP of environmental factors, intrinsic arterial and cardiopulmonary reflexes, and the influences of behavioral, emotional, and environmental factors [16]. In a high-quality review of 19 observational cohort studies and 17 clinical trial cohorts, higher long term sBP visit-to-visit variability in office settings was associated with higher: all-cause mortality (hazard ratio 1.15, 95\% confidence interval 1.09-1.22), cardiovascular disease mortality $(1.18,1.09-1.28)$, cardiovascular disease events $(1.18,1.07-1.30)$, coronary heart disease (1.10, 1.04-1.16), and stroke (1.15, 1.04-1.27)(12). Higher daytime SBP was also associated with all cause mortality whether measured at home $(\mathrm{N}=4$ papers; hazard ratio $1.15,1.06-1.26)$ or while ambulatory $(\mathrm{N}=15$ papers; hazard ratio $1.10,1.04-1.16)$. The authors noted that the observed associations with cardiovascular disease were of similar magnitude to the recognised risk marker of hyperlipidaemia, but smaller than that of mean BP level.

In hypertensive pregnancy, there is an accelerated pattern to the BPoutcome relationship, such that the vasculature has little or no time to adapt; in chronic hypertension and pre-eclampsia, there is impaired cerebral autoregulation and in pre-eclampsia, this impairment is evident regardless of the BP level $[17,18]$. Also, despite young age, systolic hypertension (particularly when BP is very high) is important in determining stroke risk, even after very short periods of raised BP, and intracerebral haemorrhage is relatively more common than outside pregnancy. Therefore, it is possible that rapid rises and falls in BP, leading to increased visit-to-visit BP variability, might be associated with adverse outcomes (and perhaps have even more of an effect) than outside pregnancy. Understanding this could be important if women with relatively modest mean BP but increased BP variability formed a novel, at-risk group and may guide treatment choices.

We examined the relationship between pregnancy outcomes and both BP level and long-term visit-to-visit variability amongst participants in the CHIPS Trial (Control of Hypertension In Pregnancy Study, NCT01192412, https://pre-empt.bcchr.ca/treatment/CHIPS) [19].

\section{Methods}

This was a secondary exploratory analysis of data from the CHIPS trial, a non-masked, pragmatic international multicentre trial conducted in 94 centres in 15 countries [19], and approved by the Research Ethics Board at the University of British Columbia as the Co-ordinating Centre (H08-00882) and at all study sites.

As previously described, after written informed consent, women at 14 weeks and 0 days to 33 weeks and 6 days gestation with non-proteinuric chronic or gestational hypertension, elevated BP (office $\mathrm{dBP}$ of $90-105 \mathrm{mmHg}$ or $85-105 \mathrm{mmHg}$ if taking antihypertensive therapy), and a live fetus were randomised centrally (minimised by centre and type of hypertension) to 'less tight' (target dBP of $100 \mathrm{~mm} \mathrm{Hg}$ ) or 'tight' control (target dBP $85 \mathrm{~mm} \mathrm{Hg}$ ) until delivery [19]. Although sBP and $\mathrm{dBP}$ were recorded in the patient diary, the CHIPS intervention was based on the dBP, unless sBP were $\geq 160 \mathrm{mmHg}$ at which point antihypertensive therapy was to be administered, regardless of the allocated group and regardless of the $\mathrm{dBP}$. Labetalol was the antihypertensive of first choice; angiotensin converting enzyme inhibitors, angiotensin-receptor antagonists, direct renin inhibitors, and atenolol were not permitted after randomisation and before delivery (due to known safety concerns over use of these drugs in pregnancy).

\subsection{Major CHIPS outcomes}

The composite primary outcome in the CHIPS trial was perinatal pregnancy loss or high-level neonatal care (greater than normal newborn care) for $>48 \mathrm{~h}$ in the first 28 days of life. Additional outcomes for the baby were birthweight $<10$ th centile, and preterm delivery at $<34$ or $<37$ weeks.

The composite secondary outcome in CHIPS was maternal: serious maternal complications before 6 weeks postpartum or until hospital discharge, whichever was later; serious maternal complications included death, stroke, eclampsia, blindness, uncontrolled hypertension, the use of inotropic agents, pulmonary oedema, respiratory failure, myocardial ischaemia or infarction, hepatic dysfunction, hepatic haematoma or rupture, renal failure, and transfusion. Additional outcomes for the woman were severe hypertension and pre-eclampsia. Further details can be found in the CHIPS protocol (http://pre-empt.cfri.ca/ CHIPS), the main CHIPS publication [19] and Table S2.

\subsection{BP level and variability}

The BP measurement technique was standardised. BP measurement was performed at office/clinic appointments, by care providers who were instructed to have the woman rest for at least five minutes, take three BP measurements over approximately two minutes, and then record all three measurements in a patient-held diary. The average of the second and third values was taken to be the BP for that visit [20]. BP values as a hospital inpatient were not recorded in the CHIPS database based on our experience from the CHIPS pilot trial [21].

The devices used to measure BP were not standardised, but were recorded. Most women had their BP taken by aneroid device (490, 49.7\%), although automated devices $(261,26.5 \%)$ and mercury sphygmomanometers $(205,20.8 \%)$ were also used (data missing for 30 women, 3.0\%) [19].

Women were seen by their maternity care provider within four weeks after randomisation, at which time adherence to the BP management algorithm ('less tight' vs. 'tight') was assessed. Thereafter, women were seen at a frequency determined by their maternity careprovider. Data on BP measurements (and co-interventions) were abstracted from the hand-held diaries at routine contacts with the site study co-ordinator (at 14-20, 21-28, 29-33, 34-40 weeks, and at delivery).

Mean BP level (sBP and dBP) was defined as the mean of values taken at all post-randomisation office/clinic visits between randomisation and delivery, regardless of the duration of participation (and thus, the number of office visits) in the trial, as this depended on gestational age at both recruitment and delivery. As previously reported, mean BP was higher with 'less tight' $(138.8 \pm 0.5 / 89.9 \pm 0.3 \mathrm{mmHg})$ vs. 'tight' (133.1 $\pm 0.5 / 85.3 \pm 0.3 \mathrm{mmHg})$ control, from randomisation until delivery (Fig. S1), post-randomisation antihypertensive therapy taken less often before delivery in 'less tight' (73.4\%) vs. 'tight' (92.6\%) control groups [19], and labetalol the agent used most often in both groups (66.9\% in 'less tight' and $67.3 \%$ in 'tight' control).

Within-participant visit-to-visit BP variability was assessed using all office/clinic visits after randomisation until delivery, with the exception of BP at randomisation; we excluded baseline BP values as we anticipated that their inclusion would bias the 'tight' control group to 


\section{Maternal outcome}
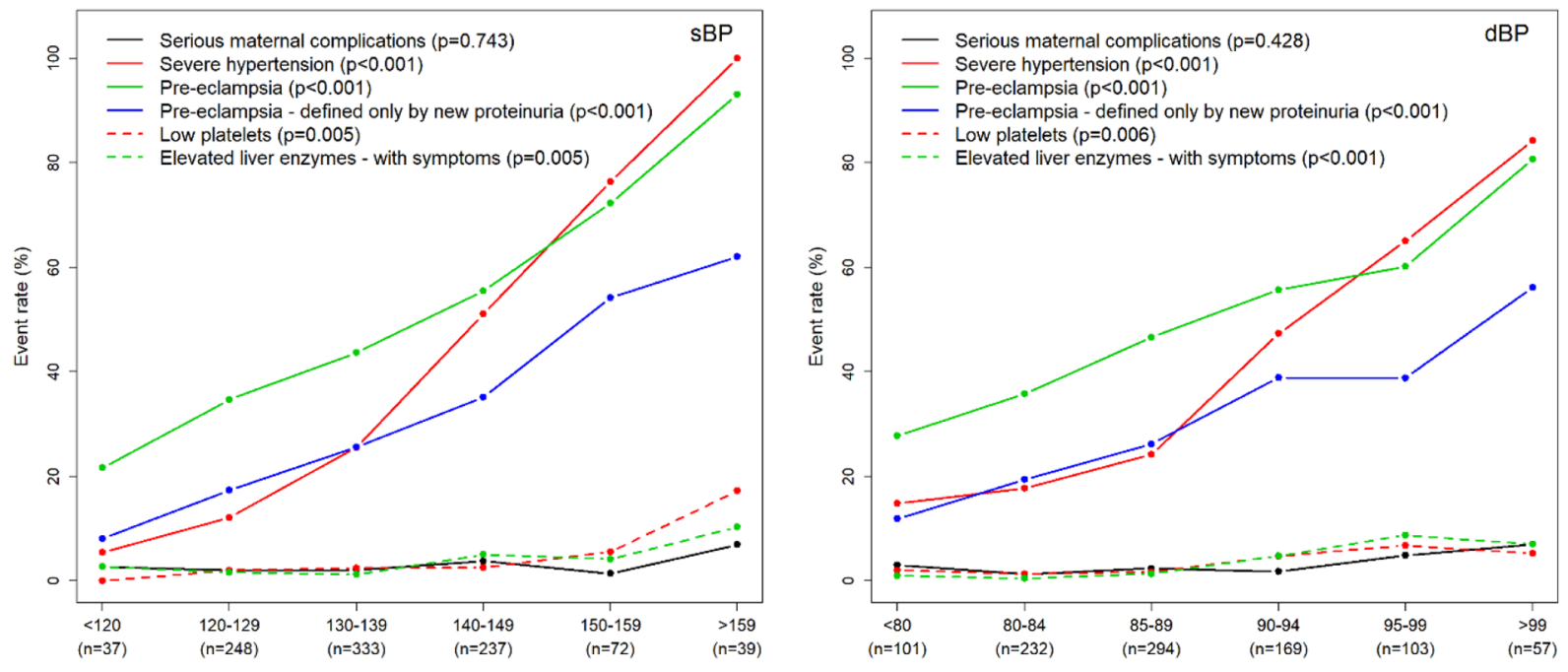

Mean sBP from randomisation to delivery $(\mathrm{mm} \mathrm{Hg})$

Mean dBP from randomisation to delivery $(\mathrm{mm} \mathrm{Hg})$

Perinatal outcome
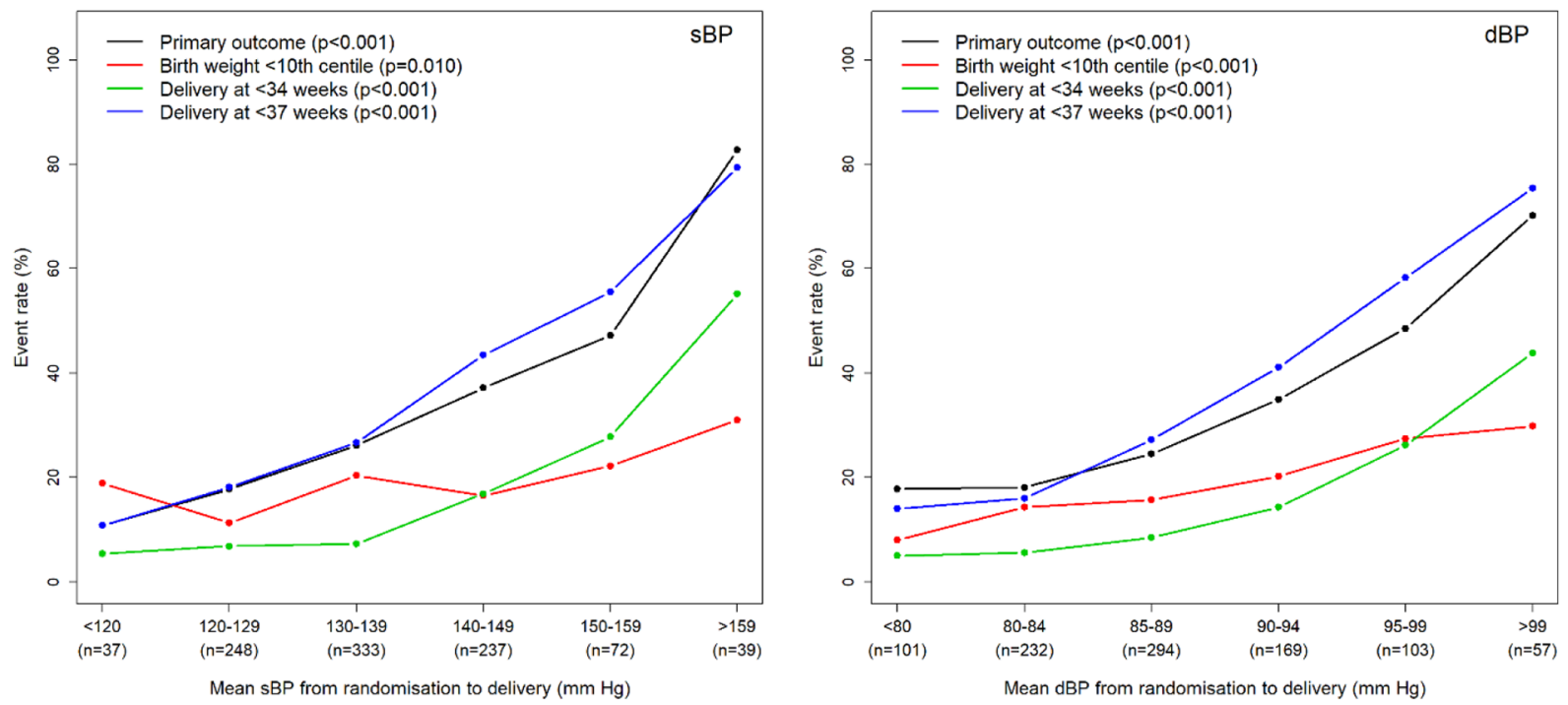

Fig. 1. Relationship between higher sBP and dBP levels and adverse maternal and perinatal outcomes*

having more BP variability as their target $\mathrm{dBP}$ required antihypertensive to be increased or a new drug started, whereas women in the 'less tight' control group would have had no change in their BP management unless dBP were higher than 100-104 mmHg (which was true for only one woman, in 'tight' control). We evaluated two traditional measures of BP variability from studies outside pregnancy: (i) within-participant standard deviation (SD) as a reflection of the dispersion of BP measurements around the mean BP level; and (ii) average real variability (ARV) as the average of the absolute successive difference of all BP values, to reflect changes that occur over short time intervals. Both measures required at least two $\mathrm{BP}$ values. We adjusted $\mathrm{BP}$ variability for mean BP level, as higher BP is associated with more BP variability. Any correlation was explored for each BP variability measure and the number of BP measurements taken.

\subsection{Analyses}

Relationships were explored between both BP level and BP variability for each of the major outcomes in CHIPS: perinatal loss or high level neonatal care for $>48 \mathrm{hr}$ (primary outcome), birth weight $<$ 10th centile, delivery at $<34$ or $<37$ weeks, serious maternal complications (secondary outcome), severe maternal hypertension, and preeclampsia.

The BP level-outcome relationship was compared between

\footnotetext{
* The BP level-outcome relationship was explored by mixed effects logistic regression, adjusted for treatment group ('less tight' vs. 'tight'), gestational age at randomisation (categorical), centre (as a random effect), hypertension type, antihypertensive at randomisation (yes/no), prior severe hypertension in this pregnancy, and gestational diabetes (yes/no). As there were only fewer than 30 women with a serious maternal complication, low platelets, or elevated liver enzymes with symptoms, centre was not included as an adjustment variable and logistic regression with Firth correction was employed for these outcomes. All analyses for severe hypertension, pre-eclampsia, primary outcome, and delivery at $<34$ and $<37$ weeks were significant without interaction with 'less tight' or 'tight' control, at $\mathrm{p}<0.001$; exceptions were serious maternal complications (not significant), low platelets (for $\mathrm{sBP}, \mathrm{p}=0.029$ for 'less tight' and $\mathrm{p}=0.050$ for 'tight' control, and for $\mathrm{dBP}, \mathrm{p}=0.09$ 'less tight' and $\mathrm{p}=0.008$ for 'tight' control), elevated liver enzymes with symptoms (for sBP, $p=0.050$ for 'less tight' and $\mathrm{p}=0.020$ for 'tight' control, and for $\mathrm{dBP}, \mathrm{p}<0.005$ for both 'less tight' and 'tight' control), and birthweight $<10$ th centile $(\mathrm{p}=0.009$ for sBP
} 
treatment groups by mixed effects logistic regression with participantspecific random intercept. Adjustment was made for treatment group ('less tight' vs. 'tight'), gestational age at randomisation (categorical), centre (as a random effect), hypertension type, antihypertensive at randomisation (yes/no), prior severe hypertension in this pregnancy, and gestational diabetes (yes/no); there were only 28 women with a serious maternal complication, so for this outcome, centre was not included as an adjustment variable and logistic regression with Firth correction was employed. Analyses were performed for each treatment group separately, and combined if there was no evidence of a treatment allocation-BP level interaction. The odds ratio (OR) for each outcome was calculated per $5 \mathrm{mmHg}$ increase in mean BP from randomisation until delivery.

The BP variability-outcome relationship was explored by mixed effects logistic regression, adjusted for average BP level (defined as the mean of the BP readings used to define variability, as variability increases with increasing BP levels) and the variables as described for BP level analyses (above). Also, analyses were performed for each treatment group separately, and combined if there was no evidence of a treatment allocation-BP variability interaction. The change in the scale of the OR was calculated per SD increase in each metric of BP variability in order to compare the relative importance of one measure with another. Correlation between BP variability measure and the number of BP measurements taken was assessed by Spearman correlation (r).

In sensitivity analyses, we: (i) restricted the definition of preeclampsia to development of new proteinuria; (ii) excluded BP values within $7 \mathrm{~d}, 14 \mathrm{~d}, 21 \mathrm{~d}$ and $28 \mathrm{~d}$ prior to the outcome of interest (for maternal outcomes) or delivery (for perinatal outcomes) to limit the extent to which BP variability may be an artefact of the outcomes themselves, potentially causing issues of reverse causality; and (iii) added a further adjustment for the last BP before birth, to account for BP trajectory. A p value $<0.05$ was considered of statistical and potential clinical significance. No adjustment was made for multiple comparisons.

All statistical analyses were performed using SAS 9.4 (SAS Institute Inc., Cary, NC) and R 3.2.0 (R Development Core Team, Vienna, Austria). TL had full access to the data and takes responsibility for its integrity and the data analysis.

\section{Results}

Of 987 women in the CHIPS trial, 961 (97.4\%) had at least one office/clinic visit (median 7, IQR 4, 10) following baseline, and contributed to the BP level analyses. 913 (92.5\%) women had at least two office visits (median 7, IQR 5, 11) and contributed to the BP variability analyses; most women $(735,74.5 \%)$ had at least four office visits at which BP was measured.

\subsection{BP level}

For the BP level and outcome analysis, both BP control groups were combined because there was no consistent difference between 'less tight' and 'tight' control with regards to the relationship between BP level and outcomes (see homogeneity of OR, Table S3). Higher BP levels were associated with more adverse maternal and perinatal outcomes for all but serious maternal complications, as shown graphically in Fig. 1 and numerically in Table 1 (by trial group as the BP levels were different in the 'less tight' and 'tight' control groups). For the mother, the association of higher mean BP level and more adverse outcomes appeared to have no inflexion point, down to mean sBP $<120 \mathrm{mmHg}$ and mean $\mathrm{dBP}<80 \mathrm{mmHg}$. For the baby, there was no inflexion point for: the CHIPS primary perinatal outcome or delivery at $<37$ weeks

(footnote continued)

within 'less tight' control, $\mathrm{p}=0.293$ for 'tight' control, and $\mathrm{p} \leq 0.003$ for $\mathrm{dBP}$ within 'less tight' and 'tight' control). from $\mathrm{sBP}<120 \mathrm{mHg}$ or $\mathrm{dBP}$ of $80-84 \mathrm{mHg}$; for delivery at $<34$ weeks from sBP $130-139 \mathrm{mmHg}$ or dBP $80-84 \mathrm{mmHg}$; or for birth weight < 10th centile from 140 to $149 / 85-89 \mathrm{mmHg}$.

\subsection{BP variability}

Median [IQR] within-participant BP variability appeared to be higher for SBP than $\mathrm{ABP}$, for both measures of SD and ARV (Table 2). There was no relationship between the number of $\mathrm{BP}$ measurements taken and either $\mathrm{BP}$ variability measured by $\mathrm{SD}(\mathrm{sBP} r=0.16, \mathrm{dBP}$ $r=0.15$ ) or ARV (sBP $r=0.05$, dBP $r=0.04$ ).

For the BP variability analyses, both BP control groups were combined given a lack of compelling evidence for a BP variability-treatment allocation interaction (Table S4). Fig. 2 shows that for the woman, greater BP variability (systolic and diastolic) was associated with higher odds of adverse maternal outcome, particularly severe hypertension and pre-eclampsia; this was true for both definitions of pre-eclampsia (i.e., broad and restrictive), for both SD and ARV variability measures, and for SBP and dBP. For the baby, the pattern appeared to be different. First, there was no significant relationship between higher BP variability and adverse perinatal outcomes (of the CHIPS primary perinatal outcome, birth weight $<10$ th centile, and delivery at $<34$ or $<37$ weeks), or for SD or ARV. variability measures, although the point estimates suggested a possible relationship between higher dBP variability and fewer adverse perinatal outcomes (Fig. 2.)

In sensitivity analyses with removal of BP values close to the time of maternal outcome or birth for perinatal outcomes, there was no longer a clear association between BP variability and adverse maternal outcomes, but the apparent association between more $\mathrm{dBP}$ variability and fewer adverse perinatal outcomes was strengthened (Figs. S2a to S2d). Also, adjustment for last BP before delivery did not substantially change the results; some p values that were just below 0.05 rose just above 0.05 and one became significant, but the OR point estimates for these and throughout very similar (Table S5).

\section{Discussion}

\subsection{Main findings}

In this secondary analysis of data from the CHIPS trial, we demonstrated relationships with adverse pregnancy outcomes for both mean BP level and BP variability. Higher post-randomisation BP level was associated with more adverse maternal and perinatal outcomes, with the observed association extending down to below 140/90 $\mathrm{mmHg}$ for many maternal (i.e., severe hypertension and pre-eclampsia) and perinatal outcomes (i.e., CHIPS primary perinatal outcome, birth weight $<10$ th centile, and delivery at $<34$ or $<37$ weeks). Higher BP variability assessed by SD or ARV (and adjusted for BP level) was associated with higher odds of severe hypertension and pre-eclampsia ( $\mathrm{p}<0.05$ ), but not perinatal outcomes. However, there was evidence of reverse causality of BP variability with maternal outcomes, following exclusion of BP values 7-28 days before the outcomes; in these sensitivity analyses, higher $\mathrm{dBP}$ variability was associated with lower perinatal risk.

\subsection{Interpretation}

Higher BP levels have long been associated with adverse pregnancy outcome. Although this has been primarily related to comparisons between hypertensive and normotensive women, recent cohorts have demonstrated a continuous relationship between BP level and adverse maternal outcome $[9,10]$, as we found in our analyses here. These findings underpin antenatal care, in which a key component is related to measurement of BP and detection of hypertension.

It has been well-established that as in non-pregnant individuals, BP varies over a 24-hour period in pregnancy, and patterns of BP change 
Table 1

Relationship between BP levels and major CHIPS outcomes*.

\begin{tabular}{|c|c|c|c|c|c|c|c|c|}
\hline \multirow[t]{2}{*}{ Outcome } & \multicolumn{4}{|c|}{ Relationship with sBP* } & \multicolumn{4}{|c|}{ Relationship with dBP* } \\
\hline & 'Less tight' & & 'Tight' & & 'Less tight' & & 'Tight' & \\
\hline Maternal & OR $(95 \% \mathrm{CI})$ & $\mathbf{p}$ & OR $(95 \% \mathrm{CI})$ & $\mathbf{p}$ & OR $(95 \% \mathrm{CI})$ & $\mathbf{p}$ & OR $(95 \% \mathrm{CI})$ & $\mathbf{p}$ \\
\hline Secondary outcome & $\begin{array}{l}1.16 \\
(0.93,1.44)\end{array}$ & 0.192 & $\begin{array}{l}0.80 \\
(0.57,1.12)\end{array}$ & 0.185 & $\begin{array}{l}1.32 \\
(0.97,1.81)\end{array}$ & 0.082 & $\begin{array}{l}0.72 \\
(0.44,1.17)\end{array}$ & 0.184 \\
\hline Severe hypertension & $\begin{array}{l}2.02 \\
(1.75,2.34)\end{array}$ & $<0.001$ & $\begin{array}{l}1.71 \\
(1.49,1.97)\end{array}$ & $<0.001$ & $\begin{array}{l}2.34 \\
(1.93,2.84)\end{array}$ & $<0.001$ & $\begin{array}{l}2.36 \\
(1.88,2.96)\end{array}$ & $<0.001$ \\
\hline Preeclampsia $\dagger$ & $\begin{array}{l}1.40 \\
(1.26,1.56)\end{array}$ & $<0.001$ & $\begin{array}{l}1.48 \\
(1.31,1.67)\end{array}$ & $<0.001$ & $\begin{array}{l}1.50 \\
(1.29,1.74)\end{array}$ & $<0.001$ & $\begin{array}{l}1.89 \\
(1.56,2.30)\end{array}$ & $<0.001$ \\
\hline Low platelets & $\begin{array}{l}1.25 \\
(1.02,1.52)\end{array}$ & 0.029 & $\begin{array}{l}1.30 \\
(1.00,1.71)\end{array}$ & 0.050 & $\begin{array}{l}1.28 \\
(0.96,1.71)\end{array}$ & 0.094 & $\begin{array}{l}1.62 \\
(1.14,2.31)\end{array}$ & 0.008 \\
\hline Elevated liver enzymes (with symptoms) & $\begin{array}{l}1.22 \\
(1.00,1.48)\end{array}$ & 0.050 & $\begin{array}{l}1.37 \\
(1.05,1.79)\end{array}$ & 0.020 & $\begin{array}{l}1.54 \\
(1.15,2.08)\end{array}$ & 0.004 & $\begin{array}{l}1.77 \\
(1.24,2.53)\end{array}$ & 0.002 \\
\hline \multicolumn{9}{|l|}{ Perinatal } \\
\hline Primary outcome & $\begin{array}{l}1.37 \\
(1.23,1.53)\end{array}$ & $<0.001$ & $\begin{array}{l}1.40 \\
(1.24,1.57)\end{array}$ & $<0.001$ & $\begin{array}{l}1.63 \\
(1.39,1.91)\end{array}$ & $<0.001$ & $\begin{array}{l}2.06 \\
(1.67,2.54)\end{array}$ & $<0.001$ \\
\hline Birth weight $<10$ th centile & $\begin{array}{l}1.17 \\
(1.04,1.31)\end{array}$ & 0.009 & $\begin{array}{l}1.06 \\
(0.95,1.20)\end{array}$ & 0.293 & $\begin{array}{l}1.51 \\
(1.27,1.81)\end{array}$ & $<0.001$ & $\begin{array}{l}1.31 \\
(1.10,1.57)\end{array}$ & 0.003 \\
\hline Delivery at $<34$ weeks & $\begin{array}{l}1.46 \\
(1.28,1.67)\end{array}$ & $<0.001$ & $\begin{array}{l}1.39 \\
(1.20,1.60)\end{array}$ & $<0.001$ & $\begin{array}{l}1.93 \\
(1.57,2.36)\end{array}$ & $<0.001$ & $\begin{array}{l}1.91 \\
(1.51,2.43)\end{array}$ & $<0.001$ \\
\hline Delivery at $<37$ weeks & $\begin{array}{l}1.35 \\
(1.22,1.50)\end{array}$ & $<0.001$ & $\begin{array}{l}1.49 \\
(1.32,1.68)\end{array}$ & $<0.001$ & $\begin{array}{l}1.71 \\
(1.45,2.01)\end{array}$ & $<0.001$ & $\begin{array}{l}2.64 \\
(2.08,3.35)\end{array}$ & $<0.001$ \\
\hline
\end{tabular}

dBP (diastolic blood pressure), sBP (systolic blood pressure)

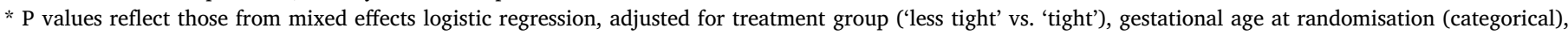

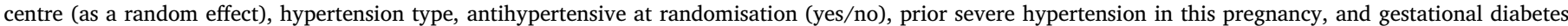

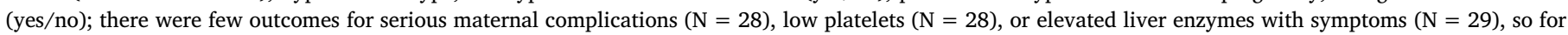

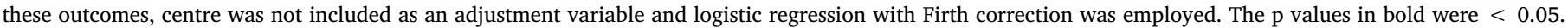
There was no evidence of a treatment allocation-BP level interaction (not presented, all p > 0.05).

$\dagger$ Defined restrictively as gestational hypertension and new proteinuria.

Table 2

BP variability for the 913 women with at least two post-randomisation office/ clinic visit in CHIPS [N (\%) or mean (SD) unless otherwise stated]

\begin{tabular}{lllll}
\hline & $\begin{array}{l}\text { Overall } \\
(\mathrm{N}=913)\end{array}$ & $\begin{array}{l}\text { 'Less tight' control } \\
(\mathrm{N}=464)\end{array}$ & $\begin{array}{l}\text { 'Tight' control } \\
(\mathrm{N}=449)\end{array}$ & p value* \\
$\begin{array}{l}\text { SD sBP } \\
\begin{array}{l}\text { Median (IQR) } \\
\text { SD dBP }\end{array}\end{array}$ & $9.0(6.7,11.6)$ & $9.2(6.8,12.1)$ & $8.7(6.7,11.3)$ & 0.165 \\
$\begin{array}{l}\text { Median (IQR) } \\
\text { ARV sBP }\end{array}$ & $6.6(4.7,8.7)$ & $6.6(4.8,8.9)$ & $6.6(4.6,8.4)$ & 0.404 \\
$\begin{array}{l}\text { Median (IQR) } \\
\text { ARV dBP }\end{array}$ & $9.2(6.8,12.1)$ & $9.2(6.9,12.3)$ & $9.3(6.7,12.1)$ & 0.824 \\
$\begin{array}{l}\text { Median (IQR) } \\
6.9(4.6,9.3)\end{array}$ & $6.9(4.7,9.2)$ & $7.0(4.5,9.3)$ & 0.881 \\
\hline
\end{tabular}

* The p values were based on Wilcoxon rank sum test.

ARV (average real variability), dBP (diastolic blood pressure), sBP (systolic blood pressure), SD (standard deviation).

are associated with onset of hypertensive disease[22]. However, we were unable to find a published analysis in hypertensive pregnancy of visit-to-visit BP variability and its relationship with outcome as reported in our study.

Our demonstration of reverse causality for severe maternal hypertension and pre-eclampsia are consistent with the diagnosis being partially responsible for the variability, as both require further rises in $\mathrm{BP}$ and higher BP contributes to higher BP variability; although we adjusted our measures of BP variability for mean BP throughout pregnancy, this would not have adjusted fully for the effect of an acute rise in BP before the outcome was diagnosed. While these are 'acute' diagnoses, our perinatal outcomes are not; rather, they reflect prolonged physiological effects over time, resulting in survival in good health (as measured by our primary perinatal outcome), good fetal growth, and the lack of an indication for iatrogenic preterm delivery. It is possible that women with higher BP variability were managed differently and that this may have improved perinatal outcome; of note, the BP variability relationship with improved perinatal outcome was related to $\mathrm{dBP}$ and CHIPS had dBP targets. Alternatively, it is possible that higher
BP variability may have resulted in better uteroplacental perfusion.

\subsection{Strengths and limitations}

The strengths of this paper relate to the quality of the CHIPS trial data, as well as the generalisability of its results given its conduct in 94 centres in 15 countries. Systolic BP level trends paralleled diastolic ones, even through the CHIPS BP targets were diastolic (i.e., $100 \mathrm{mmHg}$ for 'less tight' and $85 \mathrm{mmHg}$ for 'tight' control); importantly, no excessive lowering of BP was seen in the 'tight' control group. We used measures of BP variability from outside pregnancy and demonstrated no relationship between the frequency of BP measurement and BP variability. We have addressed many of the methodological flaws seen in BP variability literature outside pregnancy, by documenting BP measurement procedures, using $\mathrm{BP}$ values taken in the office/clinic setting and taken during the day, and adjusting BP variability for mean BP level.

Limitations include that CHIPS was a pragmatic trial and although BP measurement technique was standardised, the BP measurement devices used were not. We did not adjust for post-randomisation antihypertensive medication, as we do not have information about duration of use and dose of each agent; however, the impact of BP variability on outcome would not have been accounted for by use of labetalol or methyldopa as our previous analyses showed that the direction of the effect of these agents on outcomes was the same [23]; nifedipine, which has been associated in women with chronic hypertension with less 24hour BP variation than labetalol[24] was used by a minority of women in CHIPS. In CHIPS, we had data only on BP measurements; no biological samples were taken to allow exploration of other pathophysiological pathways that might explain the relationship between BP variability and pregnancy outcomes. We used $\mathrm{p}<0.05$ to identify associations of potential interest, but multiple comparisons were made. Finally, this was an unplanned exploratory analysis stimulated by more recent data outside pregnancy, and external validation is required to confirm the findings. 


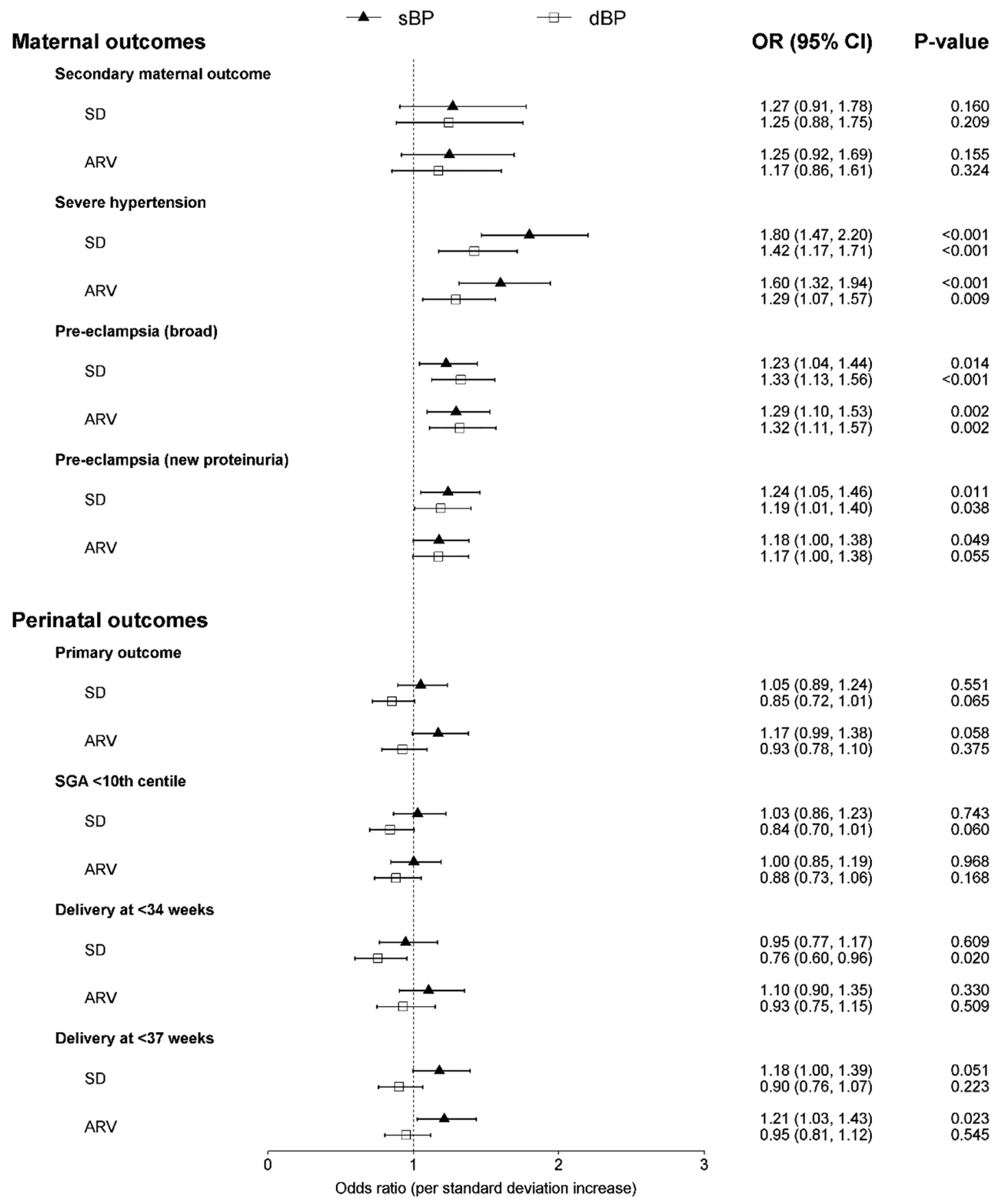

Fig. 2. Relationship between adverse outcomes and both systolic and diastolic BP variability*

\section{Conclusion}

Among hypertensive women in the CHIPS trial, higher mean BP was an adverse prognostic marker for the woman and baby, regardless of target $\mathrm{dBP}$ as in CHIPS. Independent of BP level, higher BP variability was associated with more severe hypertension and pre-eclampsia; while likely related to BP values close to the time of diagnosis, these findings suggest that when BP 'spikes' are noted, subsequent lower absolute values should not be used to negate concern even though they (particularly dBP spikes) may have been associated with better perinatal outcomes. If confirmed in future studies, it would be important to study mechanism to understand whether the relationship is mediated through physiological or iatrogenic effects, including the effects of different antihypertensive agents. In the meantime, although the data presented are exploratory and hypothesis-generating, they suggest that for the clinician, a prudent approach may be to respond to BP variability with enhanced vigilance for severe hypertension and/or pre-eclampsia, even though the impact on the baby may not be negative.

\footnotetext{
"All analyses are adjusted for treatment allocation ('less tight' vs. 'tight' control), gestational age at randomisation (categorical), centre (as a random effect), and BP level (as the mean BP at all office/clinic visits for the SD and ARV analyses). Odds ratio presented is for per unit increase in the metric. An odds ratio $>1$ suggests women with higher value of the metric have higher odds of experiencing the outcome. Please see Appendix Table S3 for the numeric values of the OR and $95 \% \mathrm{CI}$ for each comparison.
} 


\section{Acknowledgements}

We thank all the women who participated in CHIPS.

\section{Contribution to Authorship}

The secondary analyses were conceived by LAM and AGL, with active contribution by PvD, TL, and JS. TL performed the analyses. All authors participated in interpretation of the results, their interpretation and write-up, and approved the final version of the manuscript.

\section{Funding}

Supported by a grant (MCT 87522) from the Canadian Institutes of Health Research. Richard McManus and Lucy Chappell are supported by NIHR Professorships (NIHR-RP-R2-12-015 and NIHR-RP-2014-05019) and the NIHR South London and Oxford CLAHRCs (Collaborations for Leadership in Applied Health Research and Care). Laura Magee received salary support from the Michael Smith Foundation for Health Research, Vancouver, Canada and Peter von Dadelszen from the Canadian Institutes of Health Research.

\section{Disclosures}

Peter von Dadelszen reports receiving consulting fees and in-kind support of research from Alere International related to pre-eclampsia and fetal growth restriction through the provision of Triage PlGF cartridges. Richard McManus has received BP monitoring equipment for research from Omron and Lloyds Pharmacies. No other potential conflict of interest relevant to this article was reported.

\section{Appendix A. Supplementary data}

Supplementary data to this article can be found online at https:// doi.org/10.1016/j.preghy.2019.12.002.

\section{References}

[1] S. Lewington, R. Clarke, N. Qizilbash, R. Peto, R. Collins, Age-specific relevance of usual blood pressure to vascular mortality: a meta-analysis of individual data for one million adults in 61 prospective studies, Lancet 360 (9349) (2002) 1903-1913.

[2] T.W. Hansen, M. Kikuya, L. Thijs, K. Bjorklund-Bodegard, T. Kuznetsova, T. Ohkubo, et al., Prognostic superiority of daytime ambulatory over conventional blood pressure in four populations: a meta-analysis of 7,030 individuals, $\mathrm{J}$ Hypertens 25 (8) (2007) 1554-1564.

[3] P.K. Whelton, R.M. Carey, W.S. Aronow, D.E. Casey Jr., K.J. Collins, H.C. Dennison, et al., 2017 ACC/AHA/AAPA/ABC/ACPM/AGS/APhA/ASH/ASPC/NMA/PCNA Guideline for the Prevention, Detection, Evaluation, and Management of High Blood Pressure in Adults: A Report of the American College of Cardiology/American Heart Association Task Force on Clinical Practice Guidelines, J Am Coll Cardiol 71 (19) (2018) e127-e248.

[4] D. Ettehad, C.A. Emdin, A. Kiran, S.G. Anderson, T. Callender, J. Emberson, et al., Blood pressure lowering for prevention of cardiovascular disease and death: a systematic review and meta-analysis, Lancet 387 (10022) (2016) 957-967.
[5] M.R. Law, J.K. Morris, N.J. Wald, Use of blood pressure lowering drugs in the prevention of cardiovascular disease: meta-analysis of 147 randomised trials in the context of expectations from prospective epidemiological studies, BMJ 338 (2009) b1665.

[6] M.A. Brown, L.A. Magee, L.C. Kenny, S.A. Karumanchi, F.P. McCarthy, S. Saito, et al., The hypertensive disorders of pregnancy: ISSHP classification, diagnosis \& management recommendations for international practice, Pregnancy Hypertens (2018).

[7] D.A. Davey, I. MacGillivray, The classification and definition of the hypertensive disorders of pregnancy, Am J Obstet Gynecol 158 (4) (1988) 892-898.

[8] P. Stone, D. Cook, J. Hutton, G. Purdie, H. Murray, L. Harcourt, Measurements of blood pressure, oedema and proteinuria in a pregnant population of New Zealand, Aust N Z J Obstet Gynaecol 35 (1) (1995) 32-37.

[9] B.A. Payne, J.A. Hutcheon, J.M. Ansermino, D.R. Hall, Z.A. Bhutta, S.Z. Bhutta, et al., A risk prediction model for the assessment and triage of women with hypertensive disorders of pregnancy in low-resourced settings: the miniPIERS (Preeclampsia Integrated Estimate of RiSk) multi-country prospective cohort study, PLoS Med 11 (1) (2014) e1001589.

[10] P. von Dadelszen, B. Payne, J. Li, J.M. Ansermino, P.F. Broughton, A.M. Cote, et al., Prediction of adverse maternal outcomes in pre-eclampsia: development and validation of the fullPIERS model, Lancet 377 (9761) (2011) 219-227.

[11] L.A. Magee, D.P. von, J. Singer, T. Lee, E. Rey, S. Ross, et al., The CHIPS Randomized Controlled Trial (Control of Hypertension in Pregnancy Study): Is Severe Hypertension Just an Elevated Blood Pressure? Hypertension 68 (5) (2016) 1153-1159.

[12] S. Laurent, J. Cockcroft, B.L. Van, P. Boutouyrie, C. Giannattasio, D. Hayoz, et al., Expert consensus document on arterial stiffness: methodological issues and clinical applications, Eur Heart J 27 (21) (2006) 2588-2605.

[13] S. MacMahon, R. Peto, J. Cutler, R. Collins, P. Sorlie, J. Neaton, et al., Blood pressure, stroke, and coronary heart disease. Part 1, Prolonged differences in blood pressure: prospective observational studies corrected for the regression dilution bias, Lancet 335 (8692) (1990) 765-774.

[14] J.A. Staessen, J. Wang, G. Bianchi, W.H. Birkenhager, Essential hypertension, Lancet 361 (9369) (2003) 1629-1641.

[15] S.L. Stevens, S. Wood, C. Koshiaris, K. Law, P. Glasziou, R.J. Stevens, et al., Blood pressure variability and cardiovascular disease: systematic review and meta-analysis, BMJ 354 (2016) i4098.

[16] L.J. Mena, V.G. Felix, J.D. Melgarejo, G.E. Maestre, 24-Hour Blood Pressure Variability Assessed by Average Real Variability: A Systematic Review and MetaAnalysis, J Am Heart Assoc (2017) 6(10)

[17] T.R. van Veen, R.B. Panerai, S. Haeri, A.C. Griffioen, G.G. Zeeman, M.A. Belfort, Cerebral autoregulation in normal pregnancy and preeclampsia, Obstet Gynecol 122 (5) (2013) 1064-1069.

[18] T.R. van Veen, R.B. Panerai, S. Haeri, J. Singh, J.A. Adusumalli, G.G. Zeeman, et al., Cerebral autoregulation in different hypertensive disorders of pregnancy, Am J Obstet Gynecol 212 (4) (2015) 513-517.

[19] L.A. Magee, P. von Dadelszen, E. Rey, S. Ross, E. Asztalos, K.E. Murphy, et al., Lesstight versus tight control of hypertension in pregnancy, N Engl J Med 372 (5) (2015) 407-417.

[20] K.A. Nerenberg, K.B. Zarnke, A.A. Leung, K. Dasgupta, S. Butalia, K. McBrien, et al., Hypertension Canada's 2018 Guidelines for Diagnosis, Risk Assessment, Prevention, and Treatment of Hypertension in Adults and Children, Can J Cardiol 34 (5) (2018) $506-525$.

[21] L.A. Magee, P. von Dadelszen, S. Chan, A. Gafni, A. Gruslin, M. Helewa, et al., The Control of Hypertension In Pregnancy Study Pilot Trial, BJOG 114 (6) (2007) 770, e13-770, e20.

[22] D.E. Ayala, R.C. Hermida, Ambulatory blood pressure monitoring for the early identification of hypertension in pregnancy, Chronobiol Int 30 (1-2) (2013) 233-259.

[23] Magee LA, von DP, Rey E, Ross S, Asztalos E, Murphy KE et al. Do labetalol and methyldopa have different effects on pregnancy outcome? Analysis of data from the Control of Hypertension In Pregnancy Study (CHIPS) trial. BJOG 2015; (in press).

[24] E. Shawkat, H. Mistry, C. Chmiel, L. Webster, L. Chappell, E.D. Johnstone, et al., The effect of labetalol and nifedipine MR on blood pressure in women with chronic hypertension in pregnancy, Pregnancy Hypertens 11 (2018) 92-98. 